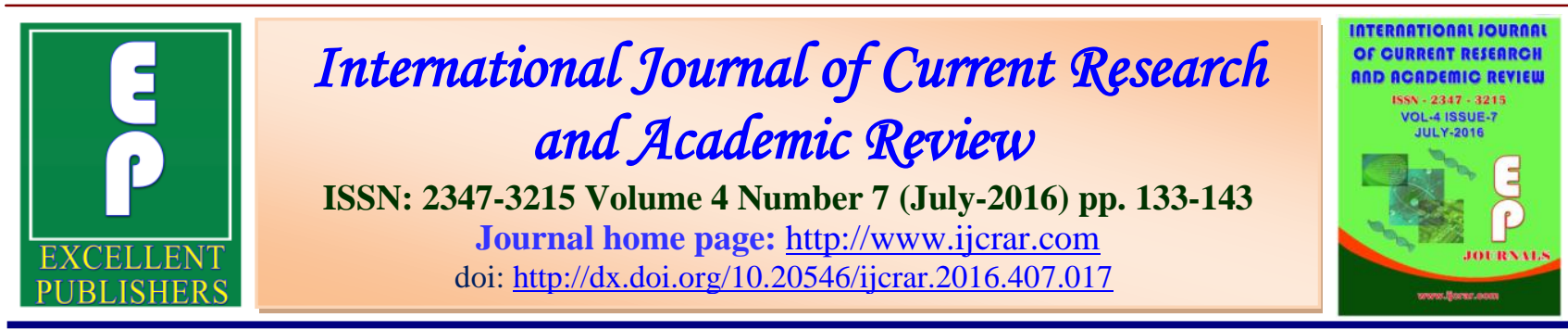

\title{
Association Between Psychiatric Disorders and Iron Deficiency Anaemia in a Tertiary Care Teaching Hospital
}

\author{
Lincy Joseph ${ }^{1}$, Mathew George ${ }^{2}$ and Elsa Ajit George ${ }^{2 *}$ \\ ${ }^{1}$ Department of Pharmaceutical Chemistry, Pushpagiri College of Pharmacy, Thiruvalla - \\ 689107, Kerala, India \\ ${ }^{2}$ Department of Pharmacology, Pushpagiri College of Pharmacy, Thiruvalla - 689107, Kerala, \\ India
}

\section{*Corresponding author}

\begin{tabular}{|c|c|}
\hline KEYWORDS & A B S T R A C $T$ \\
\hline $\begin{array}{l}\text { Iron deficiency } \\
\text { anaemia; } \\
\text { psychiatric disorders; } \\
\text { iron; } \\
\text { total iron } \\
\text { binding capacity; } \\
\text { haemoglobin; } \\
\text { medication } \\
\text { adherence. }\end{array}$ & $\begin{array}{l}\text { Iron has an important role in cognitive, behavioral, and motor development. } \\
\text { Different types of psychiatric disorders are present in the Indian population. } \\
\text { Bipolar disorder, unipolar disorder, schizophrenia, attention deficit } \\
\text { hyperactivity disorder, anxiety disorder are some of them. As there are only } \\
\text { few studies in Indian literature regarding the association between iron } \\
\text { deficiency anaemia and psychiatric disorders, the present study is undertaken. } \\
\text { This study is designed to investigate the correlation of iron deficiency } \\
\text { anaemia and psychiatric disorders and thus to find out the association } \\
\text { between psychiatric disorders and iron deficiency anaemia. Medication } \\
\text { adherence among various psychiatric patients and influence of pharmacist } \\
\text { intervention to improve medication adherence among psychiatric patients } \\
\text { were also found out in this study. }\end{array}$ \\
\hline
\end{tabular}

\section{Introduction}

Iron deficiency anemia is a common form of nutritional disorder, iron deficiency results in anemia as iron is necessary to make hemoglobin, key molecule in red blood cells responsible for the transport of oxygen. In iron deficiency anemia, the red cells appear abnormal and are unusually small (microcytic) and pale (hypochromic). The pallor of the red cells reflects their low hemoglobin content. The prevalence of iron deficiency anemia spans all ages and races. In children, iron deficiency causes developmental delays, behavioral disturbances, failure to thrive (grow) and increased infections.

Iron deficiency is a major problem in developed countries such as the US, Canada, the UK and across Europe. In developing countries, iron deficiency anemia is frequently exacerbated by malaria and worm infections. Iron deficiency causes approximately half of all anemia cases worldwide, and affects women more often 
than men. Iron-deficiency anemia affects nearly 1 billion.

Iron deficiency anaemia is characterized by a defect in hemoglobin synthesis owing to significant iron deficiency, resulting in the reduced capacity of the red blood cells to deliver oxygen to body cells and tissues, and many clinical symptoms, such as pale conjunctiva, shortness of breath, dizziness, and lethargy.

Iron deficiency anemia develops when body stores of iron drop too low to support normal red blood cell (RBC) production. Inadequate dietary iron, impaired iron absorption, bleeding, or loss of body iron in the urine may be the cause. Iron equilibrium in the body normally is regulated carefully to ensure that sufficient iron is absorbed in order to compensate for body losses of iron.

According to DSM-IV, a mental disorder is a psychological syndrome or pattern which is associated with distress (eg. via a painful symptom), disability (impairment in one or more important areas of functioning), increased risk of death, or causes a significant loss of autonomy; however it excludes normal responses such as grief from loss of a loved one, and also excludes deviant behaviour for political, religious or societal reasons not arising from a dysfunction in the individual.

The most common mental disorders that are currently prevalent in numerous countries are depression, bipolar disorder, schizophrenia, and obsessive-compulsive disorder (OCD). The dietary intake pattern of the general population in many Asian and American countries reflects that they are often deficient in many nutrients, especially essential vitamins, minerals, iron and omega-3 fatty acids. A notable feature of the diets of patients suffering from mental disorders is the severity of deficiency in these nutrients. Studies have indicated that daily supplements of vital nutrients are often effective in reducing patients' symptoms.

Iron is necessary for oxygenation and to produce energy in the cerebral parenchyma (through cytochrome oxidase), and for the synthesis of neurotransmitters and myelin. Iron deficiency is found in children with attention-deficit/hyperactivity disorder. Iron concentrations in the umbilical artery are critical during the development of the foetus, and in relation with the IQ in the child; Infantile anemia with its associated iron deficiency is associated with disturbance in the development of cognitive functions. Research findings pointed out that twice as many women as men are clinically depressed. This gender difference starts in adolescence and becomes more pronounced among married women aged 25-45, with children. Furthermore, women of childbearing age experience more depression than during other times in their lives. These indicate the possible importance of iron in the etiology of depression since its deficiency is known to cause fatigue and depression. Iron deficiency anemia is associated, forinstance, with apathy, depression, and rapid fatigue when exercising.

Different types of psychiatric disorders are present in the Indian population. Bipolar disorder, unipolar disorder, schizophrenia, attention deficit hyperactivity disorder, anxiety disorder are some of them. Some studies shown association between iron deficiency anaemia and psychiatric disorders. As there are only few studies in Indian literature regarding the association between iron deficiency anaemia and psychiatric disorders, the present study is undertaken. No similar study was done in the current study set up. This study designed 
to investigate the correlation of iron deficiency anaemia and psychiatric disorders.

Medication adherence is a growing concern to clinicians, healthcare systems, and other stakeholders (eg, payers) because of mounting evidence that nonadherence is prevalent and associated with adverse outcomes and higher costs of care. Adherence has been defined as the "active, voluntary, and collaborative involvement of the patient in a mutually acceptable course of behavior to produce a therapeutic result." This definition implies that the patient has a choice and that both patients and providers mutually establish treatment goals and the medical regimen. Medication adherence usually refers to whether patients take their medications as prescribed (eg, twice daily), as well as whether they continue to take a prescribed medication. Medication adherence behavior has thus been divided into two main concepts, namely, adherence and persistence. Although conceptually similar, adherence refers to the intensity of drug use during the duration of therapy, whereas persistence refers to the overall duration of drug therapy. Non-adherence to antipsychotic medication has a negative impact on the course of illness resulting in increased risk of relapse, rehospitalization and suicide, and increased costs to healthcare systems. Hence this study is also designed to find out medication adherence among psychiatric patients.

Mu-Hong Chen et al., (1996 - 2008) conduct a nation wide population based study on association between psychiatric disorders and iron deficiency anemia among children and adolescents. Utilizing the National Health Insurance Database from 1996 to 2008, children and adolescents with a diagnosis of IDA were identified and compared with age and gender-matched controls (1:4) in an investigation of the increased risk of psychiatric disorders. A total of 2957 patients with IDA, with an increased risk of unipolar depressive disorder $(\mathrm{OR}=2.34,95 \% \mathrm{CI}=1.58 \sim 3.46)$, bipolar disorder $\quad(\mathrm{OR}=5.78, \quad 95 \%$ $\mathrm{CI}=2.23 \sim 15.05), \quad$ anxiety disorder $(\mathrm{OR}=2.17,95 \% \mathrm{CI}=1.49 \sim 3.16)$, autism spectrum disorder $\quad(\mathrm{OR}=3.08, \quad 95 \%$ $\mathrm{CI}=1.79 \sim 5.28), \quad$ attention $\quad$ deficit hyperactivity disorder $(\mathrm{OR}=1.67,95 \%$ $\mathrm{CI}=1.29 \sim 2.17)$, tic disorder $(\mathrm{OR}=1.70$, 95\% $\mathrm{CI}=1.03 \sim 2.78$ ), developmental delay $(\mathrm{OR}=2.45, \quad 95 \% \quad \mathrm{CI}=2.00 \sim 3.00), \quad$ and mental retardation $\quad(\mathrm{OR}=2.70, \quad 95 \%$ $\mathrm{CI}=2.00 \sim 3.65$ ), were identified. A gender effect was noted, in that only female patients with IDA had an increased OR of bipolar disorder $(\mathrm{OR}=5.56,95 \% \mathrm{CI}=1.98 \sim 15.70)$ and tic disorder $\quad(\mathrm{OR}=2.95, \quad 95 \%$ $\mathrm{CI}=1.27 \sim 6.86)$. We conclude that iron deficiency increased the risk of psychiatric disorders, including mood disorders, autism spectrum disorder, attention deficit hyperactivity disorder, and developmental disorders. Further study is required to clarify the mechanism in the association between IDA and psychiatric disorder.

Sajed Faisal Al-Ali et al., (2014) conduct a study on association between autism spectrum disorder and iron deficiency in children diagnosed autism spectrum disorder in the northern west bank. 90 children with an age range of 3 to 13 years participated in a case control study distributed into study group and twocontrol groups. Thirty children diagnosed with autism according to DSMIV and ICD-10 criteria served as a study group, 30 children with mental disorders other than autism served as a control group, and 30 typically developing children taken from the public functioned as a second control group. The three groups were matched for age, gender and geographical area. Serum ferritin, 
hemoglobin, hematocrit, mean corpuscular volume, and red cell distribution width values were measured and analyzed with food habit survey and demographic data. ID was detected in $20 \%(\mathrm{~N}=6 / 30)$ of autistic children based on Serum ferritin level $(\mathrm{SF}<$ $10 \mu / 1)$,compared with $0 \%$ for the two control groups $(p=0.0001)$. Anemia was defined as hemoglobin $<110 \mathrm{~g} / \mathrm{l}$ for children under the age of 6 years and hemoglobin $<120 \mathrm{~g} / \mathrm{l}$ for children between 6 and 13 years of age. When analysis done for HGB to these six children (children who have low serum ferritin); it was found that $66.6 \%$ (4/6) of the children two were pre-school male children (HGB is less than 110g/1), and the other two were one male and one female of school children (HGB is less than $120 \mathrm{~g} / \mathrm{l}$ ) have iron deficiency anemia, and the iron deficiency anemia was $13.3 \%$ (4\30) for all autistic group. The results indicated that these differences were for males. It was found also that the frequency of low iron intake in these children was associated with feeding difficulties and food selectivity; there was a significant difference between children in the autistic group who chose foods with a red color as a favorite $23 \%(7 / 30)$ compared to the other two control groups: $0 \%$, respectively $(p=0.0001)$. The results demonstrated also a significant difference in the frequency of snacks per day $(\geq 4)$ in autistic children $40 \%$ (12/30) compared to both mental disorder $16.7 \%(\mathrm{n}=5 / 30)(\mathrm{p}=$ $0.006)$ and typically developing children $6.7 \%(\mathrm{n}=2 / 30)$ groups $(\mathrm{p}=0.001)$. Results of this study indicated that there is an association between autism, iron deficiency and anemia. Low levels of serum ferritin in autistic children might be a sign of iron deficiency and an early precursor of iron deficiency anemia. These findings suggest that food selectivity is more common in children with autism than in typically developing children. These findings suggest that ferritin levels should be measured in children with autism as a part of routine investigation.

Shruti. Srivastava et al., (2010) conduct a cross sectional study on iron profile estimation in children of behavioral disorders. The sample consisted of Forty children in the age group of 5 to 14 years suffering from behavioral disorders. Haemoglobin, Mean Corpuscular Volume, Mean Corpuscular Hemoglobin, Serum Iron,Total Iron Binding Capacity, Peripheral smear, Percentage transferrin saturation, serum ferritin estimations were done. The behavioral symptoms were scored on Achenback Child Behavior Checklist. Results: Iron deficiency anemia was present in $75 \%$ of the children with behavioral symptoms. Serum ferritin was abnormal (<20micrograms/litre) in $67.8 \%$ of the children. There was statistically significant association between pica and iron deficiency anemia $(p<0.001)$.Serum ferrittin levels correlated negatively with behavioral symptoms ( $\mathrm{r}=-.067)$, though not significant.

Rebecca J. Stoltzfus et al., conducted comprehensive reviews of published literature linking iron deficiency to disability and death for four potential outcomes: child mortality, perinatal mortality, maternal mortality and mild mental retardation. For all of these outcomes, the best available data were prospective observational studies in which anaemia or haemoglobin concentration was the risk factor. Data on child mortality were not adequate for this task, although a true risk cannot be precluded by the data. Summary relative risks for perinatal mortality (10 studies), maternal mortality (six studies) and mental retardation (fivestudies) were estimated using random effects models (both mortality outcomes) or a fixed-effects model (retardation) and weighting individual estimates by the inverse of their within- 
study variance. For mortality outcomes, the bivariate relations between haemoglobin and death were used. In two studies of perinatal mortality, unadjusted and multivariate adjusted odds ratios were compared to assess the potential degree of bias in the unadjusted associations. For mental retardation, published multivariate adjusted relations between haemoglobin and IQ were used. Global anaemia prevalence data were supplied by the World Health Organization (WHO), and converted to mean haemoglobin concentrations, assuming normal distribution and observed standard deviations from a large number of studies. To estimate the haemoglobin distribution if iron deficiency were corrected, we assumed the prevalence of anaemia in women and children would be reduced by $50 \%$. On average, for the world, this would increase haemoglobin concentration by about 0.45 $\mathrm{g} / \mathrm{dl}$ (range: $0.0 \mathrm{~g} / \mathrm{dl}$ to $1.28 \mathrm{~g} / \mathrm{dl}$ ).

After adjusting for several important covariates, the OR for mild or moderate mental retardation at school age remained significant in their model, which treated haemoglobin concentration as a continuous risk factor. This finding supports the plausibility of our assumption that the association between mean cognitive scores and anaemia is associated with increased risks of mild mental retardation. The available evidence suggests that iron deficiency anaemia contributes substantially to death and disability in the world. The great majority of this disease burden is in Africa and Asia and derives from anaemia in pregnancy and early childhood. This evidence is based on critical assumptions, most importantly, that the observed prospective relationships are causal in nature, and that the relationships analysed using anaemia as the risk factor pertain equally to iron deficiency anaemia as one particular form of anaemia.
Jyoti Batra and Archana Sood conduct a review on iron deficiency anaemia : effect on congnitive development in children. Studies has indicated that anemic children of less than 2 years have failed to catch up with non anemic children even after iron supplementation. Anemic children of more than 2 years also usually had poorer cognition and school achievements as compared to non-anemic once. They usually catch up in cognition with repeated testing and treatment but not in school achievement. Iron deficiency identifies children at concurrent and future risk of poor development. It is also concluded that iron deficiency is usually associated with many psychosocial, economic and biomedical disadvantages. The iron deficiency during developmental stages of brain (i.e. fetus) may also cause irreversible disturbances and damages to GABA neurotransmitter system. Most of the co-relational and experimental studies done earlier confirmed the hypothesis that iron deficiency of mild to moderate nature has an adverse effect on congnitive development. Therefore, it may be logical to suggest that impairment of higher mental function like cognition and learning in humans may be linked to changes in neurotransmitter receptors and consequent signal transduction process in the nervous system.

Suying Chang et al., (2002-2006) conducted a study on effect of iron deficiency anemia in pregnancy on child mental development in rural china. The aim of this study was to determine the impact of iron deficiency anemia (IDA) in pregnancy on young child development. A 2-year follow-up of 850 children born to women who participated in a double-blind cluster randomized controlled trial of prenatal micronutrient supplementation in western rural China. These women were randomly assigned to receive either daily folic acid, 
iron/folic acid (60 mg iron), or multiple micronutrients (with $30 \mathrm{mg}$ iron) during pregnancy. Children were categorized into the prenatal-IDA and prenatal-non-IDA groups based on the mother's hemoglobin in the third trimester. Each group contained 3 subgroups based on mother's treatment: folic acid, iron/folic acid, and multiple micronutrients. Bayley scales of infant development were administered to the children to assess their development at 3,6, 12, 18, and 24 months of age. Compared with the prenatal-non-IDA group, the prenatal-IDA group showed a significantly lower mental development index at 12, 18, and 24 months of age. The adjusted mean difference was 5.8 (95\% confidence interval [CI], 1.1-10.5), 5.1 (95\% CI, 1.2-9.0), and 5.3 (95\% CI, 0.9-9.7), respectively. Further analysis showed that the mental development indexes in the prenatal-IDA group and prenatal-non- IDA group were similar with supplementation of iron/folic acid but were significantly lower in the prenatal-IDA group with supplementation of folic acid or multiple micronutrients. The study concluded that prenatal IDA in the third trimester is associated with mental development of the child. However, prenatal supplementation with sufficient iron protects child development even when the woman's IDA was not properly corrected in pregnancy.

\section{Materials and Methods}

This prospective experimental study was took place in Pushpagiri Medical College, Thiruvalla among Psychiatry patients. In this study 50 subjects were involved. Their age was between 17 to 85 years with an average age of 40.70 years for both sexes. Inclusion criteria were psychiatric patients having bipolar disorder, schizophrenia, depression and anxiety. Those who give consent voluntarily to participate in the study. Both male and female. Exclusion criteria were Patients who are not willing to give consent. Patients having other comorbidities. The objectives of study were: to differentiate the prevalence of iron deficiency anaemia in various psychiatric disorders such as bipolar disorder, unipolar depression, schizophrenia, anxiety disorder, to find out the age group and gender having greater association between psychiatric disorders and iron deficiency anaemia, to find out the food habits among the patients, to find out the medication adherence among patients with psychiatric disorders. The instruments of study were: Semi auto analyser, MARS medication adherence scale, patient information data collection form.

\section{Experimental Procedure}

The study was carried out after getting approval from Institutional Ethics Committee. All patients were given a brief introduction regarding the study and the confidentiality of data. A written informed consent was obtained from the patient or care giver. Patients diagnosed with psychiatric disorders such as bipolar disorder, unipolar depression, schizophrenia, anxiety disorder were selected for the study. 50 patients were included in the study. The collected residual blood were analysed for iron and total iron binding capacity (TIBC) using semi auto analyser in the Pushpagiri College of Pharmacy. Medication adherence of the patients were found out using MARS medication adherence scale.

\section{Statistical Analysis}

All statistical analyzes were performed using SPSS software. Mean and standard deviation were used for the variables of the study. To determine the association between psychiatric disorders, age, gender and diet with iron deficiency anaemia chi square test were used. The significant association 
between the variables under consideration were found out if $\mathrm{p}$ value is less than 0.05 .

\section{Results and Discussion}

A total of 50 psychiatric patients were included in this study. Among these 26 $(52.0 \%)$ were having bipolar disorder, 16 $(32.0 \%)$ were having schizophrenia, 7 $(14.0 \%)$ were having depression and 1 (2.0\%) have anxiety. 22 patients $(44.0 \%)$ were male and 28 patients $(56.0 \%)$ were female (table.1) (graph.1). Among these 3 $(6.0 \%)$ male patients were having iron deficiency anaemia and $8(16.0 \%)$ female patients were having iron deficiency anaemia. (table 2)(graph 2) These were studied and no significant differences were observed.

Type of diet were studied; all patients were having mixed diet and $34(68.0 \%)$ patients were having a rich diet where as $16(32.0 \%)$ patients were having a poor diet. $11(22.0 \%)$ patients having iron deficiency anaemia were found to have a poor diet. These were studied and significant differences were observed between the type of diet of these patients having psychiatric disorder with iron deficiency anaemia (table 3) (graph 3). $\mathrm{P}$ value was found to be less than 0.05 .

Table.1 Table showing patients with psychiatric disorders and iron deficiency anaemia

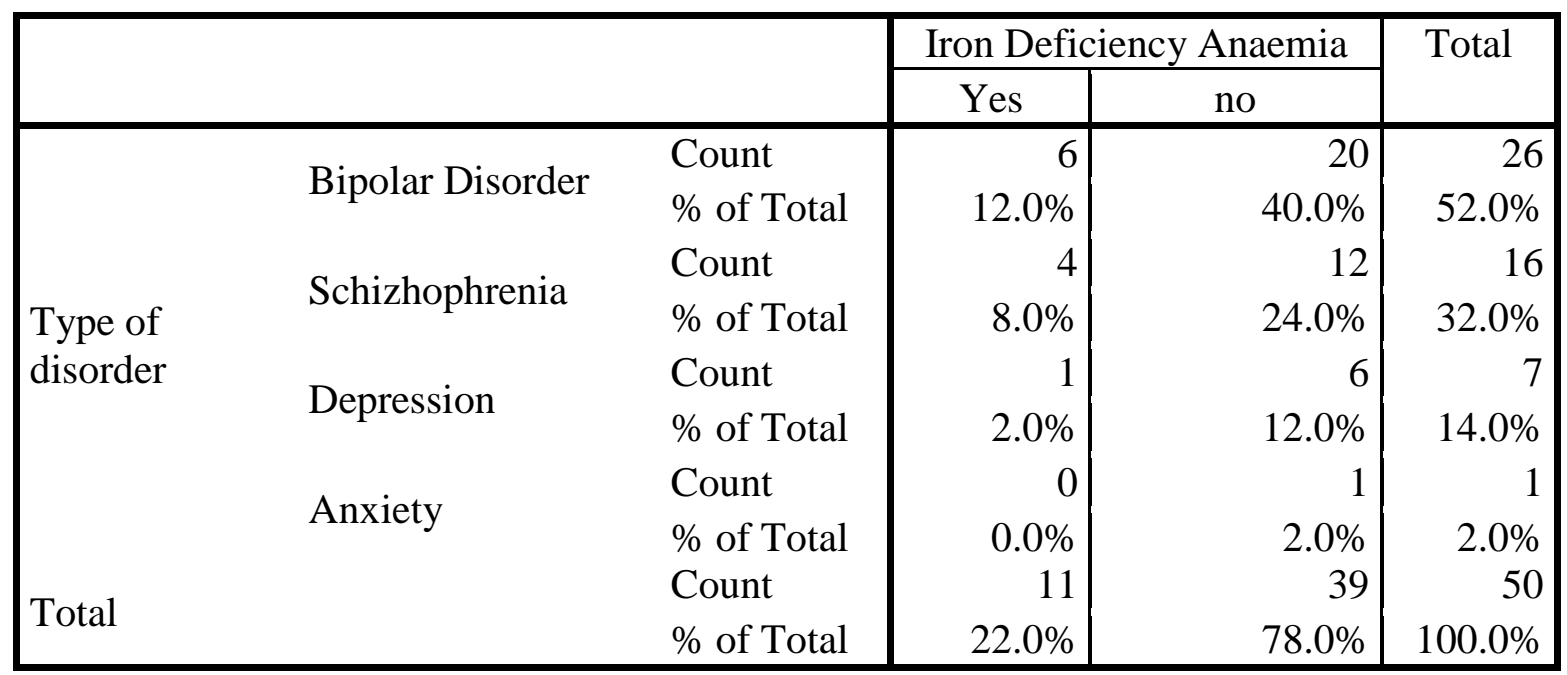

Table.2 Table showing relation with sex and iron deficiency anaemia

\begin{tabular}{|c|c|c|c|c|c|}
\hline \multicolumn{6}{|c|}{ Crosstab } \\
\hline & & & \multicolumn{2}{|c|}{ Iron Deficiency Anaemia } & \multirow[t]{2}{*}{ Total } \\
\hline & & & yes & No & \\
\hline \multirow{4}{*}{$\operatorname{sex}$} & \multirow{2}{*}{ MALE } & Count & 3 & 19 & 22 \\
\hline & & $\%$ of Total & $6.0 \%$ & $38.0 \%$ & $44.0 \%$ \\
\hline & \multirow{2}{*}{$\begin{array}{l}\text { FEMAL } \\
\text { E }\end{array}$} & Count & 8 & 20 & 28 \\
\hline & & $\%$ of Total & $16.0 \%$ & $40.0 \%$ & $56.0 \%$ \\
\hline \multirow{2}{*}{\multicolumn{2}{|c|}{ Total }} & Count & 11 & 39 & 50 \\
\hline & & $\%$ of Total & $22.0 \%$ & $78.0 \%$ & $100.0 \%$ \\
\hline
\end{tabular}


Int.J.Curr.Res.Aca.Rev.2016; 4(7): 133-143

Table.3 Table showing relation with type of diet with iron deficiency anaemia

\begin{tabular}{|c|c|c|c|c|}
\hline & \multicolumn{2}{|c|}{$\begin{array}{c}\text { Iron Deficiency } \\
\text { Anaemia }\end{array}$} & \multirow[t]{2}{*}{ Total } \\
\hline & & yes & no & \\
\hline \multirow{4}{*}{ Type of Diet } & Count & 0 & 34 & 34 \\
\hline & $\%$ of Total & $0.0 \%$ & $68.0 \%$ & $68.0 \%$ \\
\hline & Count & 11 & 5 & 16 \\
\hline & $\%$ of Total & $22.0 \%$ & $10.0 \%$ & $32.0 \%$ \\
\hline \multirow{2}{*}{ Total } & Count & 11 & 39 & 50 \\
\hline & $\%$ of Total & $22.0 \%$ & $78.0 \%$ & $100.0 \%$ \\
\hline
\end{tabular}

Table.4 Medication adherence before and after pharmacist intervention

\begin{tabular}{|lll|r|r|r|}
\hline & & & \multicolumn{2}{|c|}{ After intervention } & \multirow{2}{*}{ Total } \\
\cline { 4 - 5 } & & & Yes & \multicolumn{1}{c|}{ no } & \\
\hline \multirow{3}{*}{ Before intervention } & yes & Count & 6 & 0 & 6 \\
& & \% of Total & $12.0 \%$ & $0.0 \%$ & $12.0 \%$ \\
& no & Count & 14 & 30 & 44 \\
\multirow{2}{*}{ Total } & & \% of Total & $28.0 \%$ & $60.0 \%$ & $88.0 \%$ \\
& & Count & 20 & 30 & 50 \\
& & \% of Total & $40.0 \%$ & $60.0 \%$ & $100.0 \%$ \\
\hline
\end{tabular}

Graph.1

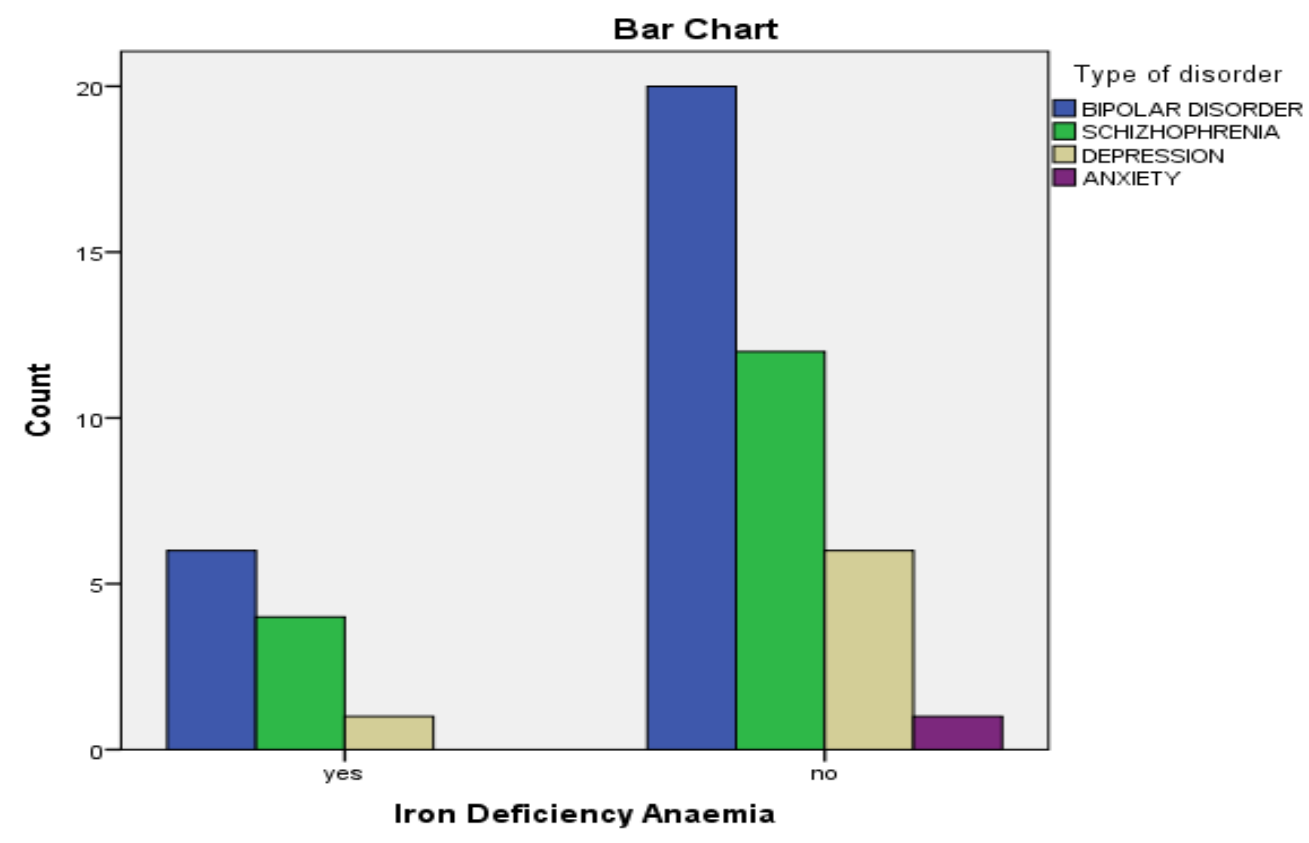


Int.J.Curr.Res.Aca.Rev.2016; 4(7): 133-143

Graph.2

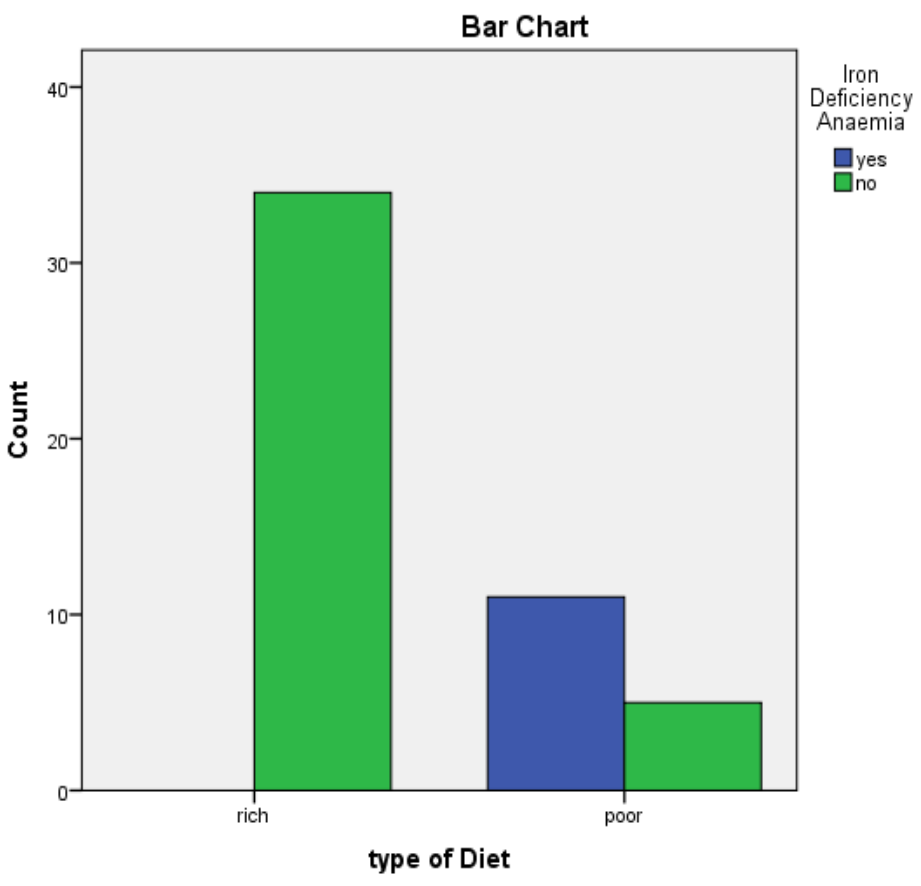

Graph 3

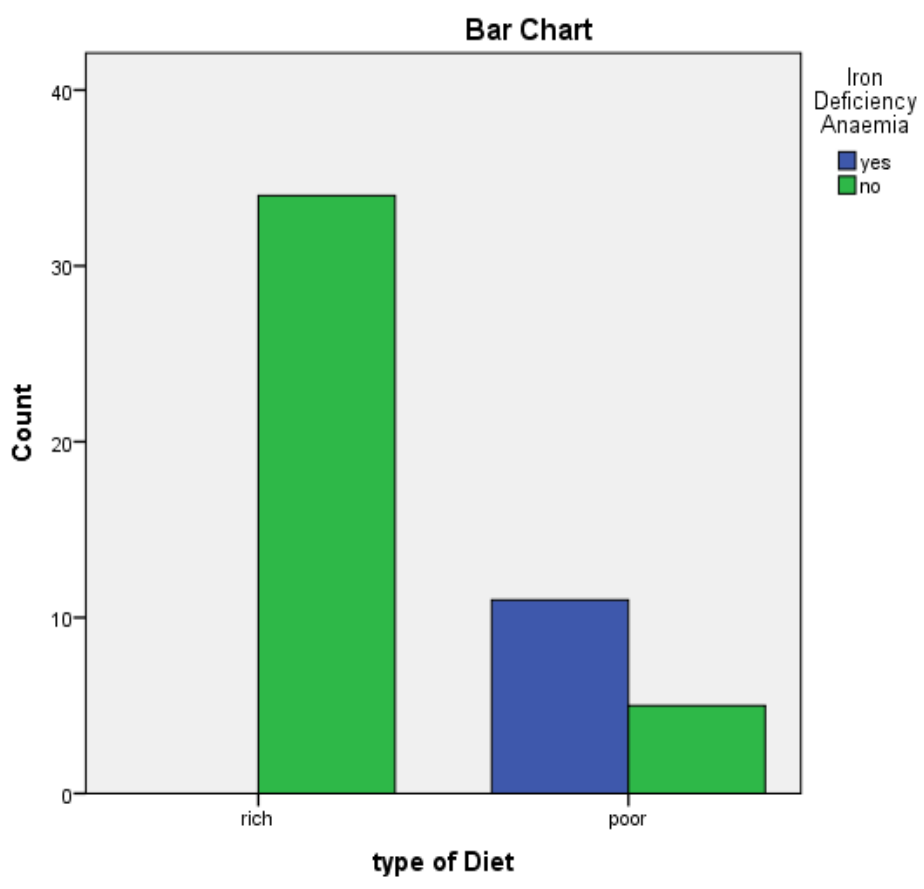




\section{Graph.4}

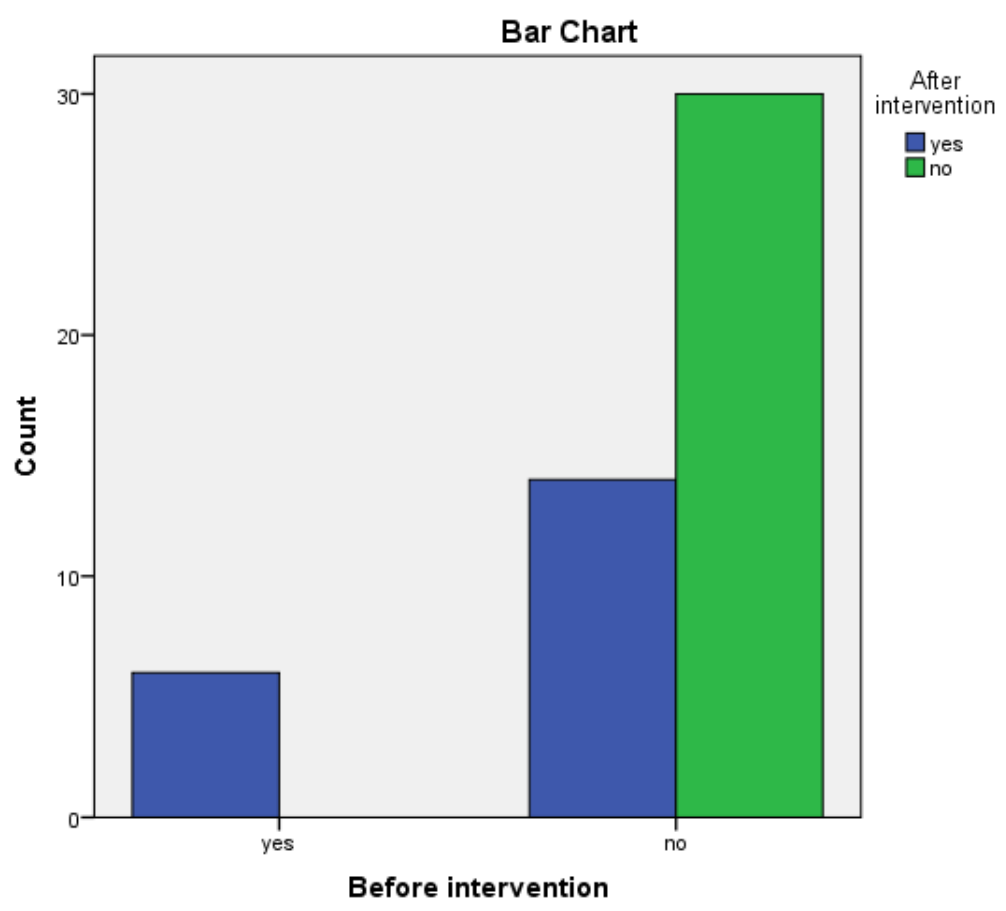

Medication adherence of these 50 psychiatric patients were studied using 'MARS' medication adherence rating scale. Initially $6(12.0 \%)$ patients were found to be adherent to medication then after pharmacist intervention $14(28.0 \%)$ more patients were found to be adherent to medication (table 4) (graph 4). These were studied and statistically significant value $(\mathrm{p}<0.05)$ was obtained.

In conclusion, this study do not find out direct correlation between psychiatric disorders and iron deficiency anaemia among the psychiatric patients in pushpagiri hospital; whereas an association was found out in relation to diet of the patients. Poor diet, so due to psychiatric disorders tend to increase the occurrence of iron deficiency anaemia. Medication adherence among the patients were found to be low and pharmacist intervention improved medication adherence among patients. It is found that counselling the bystander of patient is also very important to improve medication adherence.

\section{References}

Joseph T Dipiro, Robert L. Talbert, Gary C. Yee, Gary R. Matzke, Barbara G. Wells, L. Michael Posey. Anaemias. Pharmacotherapy:

A

Pathophysiological Approach. 2005, 6:1805.

Jyoti Batra and Archana Sood. Iron Deficiency Anaemia : Effect On Congnitive Development In Children : A Review. Indian Journal of Clinical Biochemistry. 2005, 20 (2): 119-125.

Mu-Hong Chen, Tung-Ping Su, Ying-Sheue Chen, Ju-Wei Hsu, Kai-Lin Huang, Wen-Han Chang, Tzeng-Ji Chen and Ya-Mei Bai. Association between psychiatric disorders and iron deficiency anemia among children and adolescents: a nationwide populationbased study. BioMedCentral Psychiatry 2013, 13:161. 
Rebecca J. Stoltzfus, Luke Mullany and Robert E. Black. Iron Deficiency Anaemia. Comparative Quantification of Health Risks. 163-209.

Sajed Faisal Al-Ali MSN, Dr. Sabrina Russo $\mathrm{PhD}$, Dr. Aidah Alkaissi, PhD. Association between Autism Spectrum Disorder and Iron Deficiency in Children Diagnosed Autism Spectrum Disorder in the Northern West Bank. Journal of Health, Medicine and Nursing 2015, 16:2-10.

Shruti. Srivastava, Bhatia MS, Rusia U, Rusia A. Iron Profile Estimation in
Children of Behavioral Disorders. Delhi Psychiatry Journal. 2010. 13(2):339-340.

Suying Chang, Lingxia Zen, Inge D. Brouwer, Frans J. Kok, and Hong Yan. Paediatrics. 2013. 131 (3). 755 763.

Thach Duc Tran, Beverley-Ann Biggs, Tuan Tran, Gerard J. Casey, Sarah Hanieh, Julie Anne Simpson, Terence Dwyer, Jane Fisher. Iron deficiency anaemia in pregnant women. Plos One. 2013. 8(10). $2-10$.

\section{How to cite this article:}

Lincy Joseph, Mathew George and Elsa Ajit George. 2016. Association Between Psychiatric Disorders and Iron Deficiency Anaemia in a Tertiary Care Teaching Hospital. Int.J.Curr.Res.Aca.Rev.4 (7): 133-143. doi: http://dx.doi.org/10.20546/ijcrar.2016.407.017 